\title{
Viewpoint
}

\section{A new phase for ytterbium atoms}

\author{
Henning Moritz and Tilman Esslinger \\ Institute for Quantum Electronics, ETH Zurich, 8093-Zurich, Switzerland \\ Published April 20, 2009 \\ In a cooled and trapped cloud of ytterbium atoms, the transition from a superfluid to an insulating state has been \\ observed, opening up new possibilities for precision measurements, optical clocks, and quantum computing.
}

Subject Areas: Atomic and Molecular Physics

\begin{abstract}
A Viewpoint on:
Mott insulator of ultracold alkaline-earth-metal-like atoms

Takeshi Fukuhara, Seiji Sugawa, Masahito Sugimoto, Shintaro Taie and Yoshiro Takahashi

Phys. Rev. A 79, 041604 (2009) - Published April 20, 2009
\end{abstract}

The cooling and trapping of atoms is one of the impressive success stories in contemporary physics. After all, the achievement of Bose-Einstein condensation has not just been the finale to a tremendous effort but also the starting point for a new chapter in many-body physics. The research now encompasses a diverse range of topics including strongly correlated systems, molecular and fermionic superfluids, as well as quantum computation and precision measurements. Yet most of the experiments have been performed with alkali atoms. Now, progress made with elements containing two electrons in their outer shell, such as helium or ytterbium (which could be called "alkaline-earth-like" in analogy to the two-electron alkaline-earth elements such as beryllium) is coming to fruition. In Physical Review A, T. Fukuhura, S. Sugawa, M. Sugimoto, S. Taie, and Y. Takahashi of Kyoto University, Japan, report their observation of the transition from a superfluid to a Mott insulating phase in a quantum degenerate gas of ytterbium atoms [1]. The transition has already been observed with ultracold alkali atoms, but given the unique spectroscopic properties of ytterbium and its cousins, exciting new prospects for quantum computing and highprecision metrology are now within reach.

Ideally, a quantum computer-and also an atomic clock-should consist of a large number of well-isolated two-level systems with long coherence times. In the case of a quantum computer these qubits should also be addressable and offer the possibility of establishing pairwise interactions to perform local and two-qubit operations. At first it seems bizarre to envisage for this task a cloud of atoms with its particles moving around in random directions. Even at the ultralow temperatures present in a Bose-Einstein condensate, where the atomic motion is reduced to a minimum, the atoms are delocalized and cannot be addressed.

The solution to this apparent impossibility came from an unexpected direction, namely from quantum many-

DOI: $10.1103 /$ Physics.2.31

URL: http://link.aps.org/doi/10.1103/Physics.2.31 body physics. In 1998, Dieter Jaksch and co-workers [2] recognized that it should be possible to realize so-called Hubbard models with ultracold atoms. Hubbard models are well known in condensed matter physics and are used to describe electronic properties of materials. The model assumes that particles hop on a static lattice and experience only local on-site interactions. The bosonic version of this model was originally studied by Fisher et al.[3], who predicted a quantum phase transition from a superfluid to a Mott insulating state. In the Mott state each particle is localized to a lattice site, making it, in principle, addressable. Indeed, by loading a Bose-Einstein condensate of rubidium atoms into an optical lattice, formed by interfering laser beams, Greiner et al.[4] were able to observe the transition from a superfluid to a Mott insulating state.

In earlier work, the Kyoto University team of Takasu et al. pioneered Bose-Einstein condensation in a cloud of ytterbium atoms [5]. In order to observe the transition of the superfluid to a Mott insulting phase, Fukuhara and co-workers now placed the trapped ytterbium condensate at the crossing point of three mutually perpendicular laser standing waves (Fig. 1). This so-called optical lattice creates a periodic potential for the atoms that is of simple cubic symmetry and is superposed to the harmonic confinement of the trap. The authors smoothly increased the strength of the optical lattice potential to various levels before suddenly switching it off simultaneously with the trap. After $10 \mathrm{~ms}$ of ballistic expansion, they illuminated the cloud with a pulse of resonant laser light and recorded the absorption image. When releasing the atoms from a weak optical lattice, they observed a matter-wave interference pattern analogous to the diffraction pattern seen when illuminating a grating with laser light. This is expected for the delocalized wave function of a Bose-Einstein condensed state. Its phase coherence extends over the entire condensate and the observed interference pattern reflects the spatial

(C) 2009 American Physical Society 


\section{Phys̄îs}

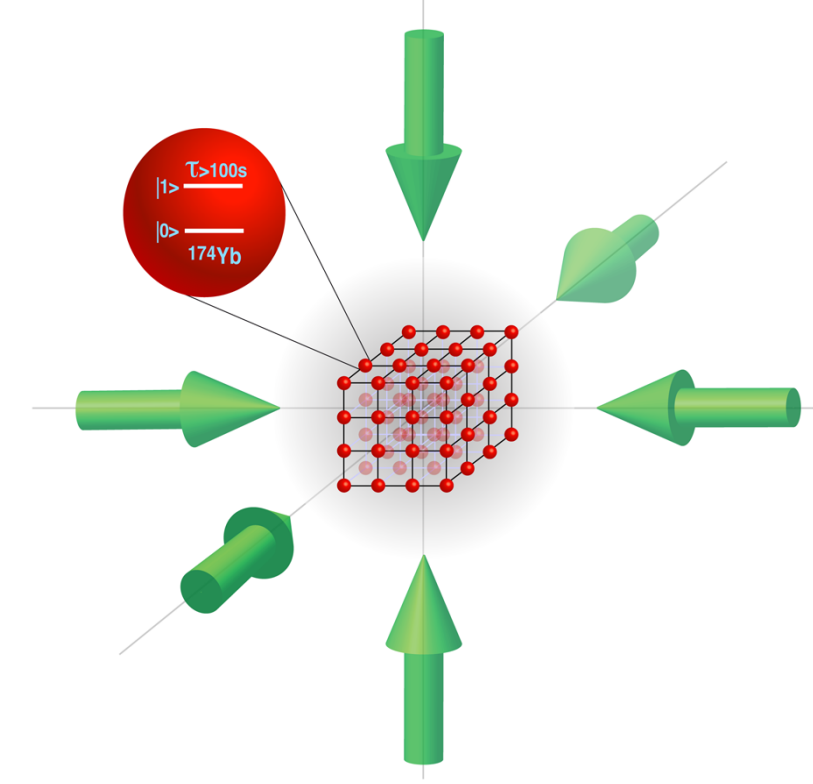

FIG. 1: A Mott insulator of ytterbium atoms. Three pairs of counterpropagating laser beams trap the atoms in a Mott insulating state of cubic geometry. The atoms are localized to lattice sites due to the strong mutual repulsion. In this configuration, each ytterbium atom can be envisaged as a qubit. (Illustration: Alan Stonebraker)

symmetry of the periodic lattice potential, just as for the optical analogue.

For deeper lattice potentials, the authors observed a disappearance of the interference pattern, which is a signature of the transition to a Mott insulating state. This transition is governed by the competition between kinetic and interaction energy with the gas seeking to minimize its total energy. At low lattice depth, kinetic energy prevails and is minimized by delocalization. As a consequence, the atom number on each lattice site fluctuates. Increasing the lattice depth reduces tunneling and flattens the lowest energy band. This diminishes the role of kinetic energy and on entering the Mott insulator the repulsive on-site interaction becomes the dominant energy scale. The atoms start to localize in order to avoid interactions. This minimizes the energy penalty associated with quantum fluctuations of the atom number. As a consequence, the long-range phase coherence vanishes, which is observed in the experiment.

Having prepared a Mott insulating state one can now think of the atoms as qubits. Each one resides on a different lattice site and is well isolated from the environment. The distinctive advantage of alkaline-earth atoms is their protection against decoherence caused by omnipresent magnetic field fluctuations. In contrast to alkali atoms with a single outer electron, the two outer electrons of ytterbium can pair up to a total electronic spin of zero, resulting in a vanishing magnetic moment. A valuable attribute is the existence of a metastable excited state with a lifetime in excess of $100 \mathrm{~s}[6]$. In this excited state, the electron spins add up to a total spin of one, making the transition to the spin-zero ground state forbidden. Even this state is magnetic-field-insensitive because the total angular moment and spin of the electrons cancel.

Considered as a qubit these two states have another exciting property. It is possible to choose laser frequencies such that they induce optical potentials either only for the ground state or only for the excited state $[7,8]$. This may allow the logical zero to be spatially separated from the logical one, represented by the ground and excited state, respectively. For example, a qubit can be shifted to its left neighbor, interact with it and be brought back to its initial position, all conditional on its being in the excited state $[8,9]$. This way, a two-qubit gate can be realized [10]. These and further features make alkaline-earth-like atoms such as ytterbium particularly attractive for quantum computation and simulation, as outlined in recent proposals [8] containing further concepts for fermionic isotopes.

The requirements for quantum computing and highprecision metrology often coincide and alkaline-earthlike atoms are considered to be ideal candidates for optical clocks. The extremely narrow optical transitions to the metastable states and the magnetic field insensitivity are ideally suited for ultrahigh precision spectroscopy. Indeed, optical lattice clocks with strontium [11] and ytterbium [12] have been realized with a fractional frequency uncertainty of down to $10^{-16}[13]$. In these clocks the atoms are trapped in single-beam optical lattices to avoid Doppler broadening caused by the atomic motion. One of the main limiting factors in present experiments is the clock shift arising from the collisional interaction between atoms. It is here that the realization of Mott insulating state may have an immediate impact. In a Mott insulating state with one atom per site, the collisional shift is strongly suppressed. Perhaps, in the not too distant future, the standard of timekeeping will be dramatically improved by the many-body physics of a Mott insulator.

\section{References}

[1] T. Fukuhara, S. Sugawa, M. Sugimoto, S. Taie, and Y. Takahashi, Phys. Rev. A 79, 041604 (2009).

[2] D. Jaksch, C. Bruder, J. I. Cirac, C. W. Gardiner, and P. Zoller, Phys. Rev. Lett. 81, 3108 (1998).

[3] M. P. A. Fisher, P. B. Weichmann, G. Grinstein, and D. S. Fisher, Phys. Rev. B 40, 546 (1989).

[4] M. Greiner, O. Mandel, T. Esslinger, T. W. Hänsch, and I. Bloch, Nature 415, 39 (2002).

[5] Y. Takasu, K. Maki, K. Komori, T. Takano, K. Honda, M. Kumakura, T. Yabuzaki, and Y. Takahashi, Phys. Rev. Lett. 91, 040404 (2003).

[6] A. V. Taichenachev, V. I. Yudin, C. W. Oates, C. W. Hoyt, Z. W. Barber, and L. Hollberg, Phys. Rev. Lett. 96, 083001 (2006).

[7] J. Ye, H.J. Kimble, and H. Katori, Science 320, 1734 (2008). 
[8] A. J. Daley, M. M. Boyd, J. Ye, and P. Zoller, Phys. Rev. Lett. 101, 170504 (2008); A.V. Gorshkov, A. M. Rey, A. J. Daley, M. M. Boyd, J. Ye, P. Zoller, and M. D. Lukin, Phys. Rev. Lett. 99, 110503 (2009).

[9] D. Jaksch, H.-J. Briegel, J. I. Cirac, C. W. Gardiner, and P. Zoller, Phys. Rev. Lett. 102, 1975 (1999); O. Mandel, M. Greiner, A. Widera, T. Rom, T. W. Hänsch, and I. Bloch, Phys. Rev. Lett. 91, 010407 (2003).

[10] D. Hayes, P. Julienne, and I. H. Deutsch, Phys. Rev. Lett. 98, 070501 (2007); M. Anderlini, P. J. Lee, B. L. Brown, J. Sebby-Strabley, W. D. Phillips, and J. V. Porto, Nature 448, 452 (2007); S. Trotzky, P. Cheinet, S. Fölling, M. Feld, U. Schnorrberger, A. M. Rey, A.
Polkovnikov, E. A. Demler, M. D. Lukin, and I. Bloch, Science 319, 295 (2008).

[11] M. Takamoto, F.-L. Hong, R. Higashi, and H. Katori, Nature 435, 321 (2005); A. D. Ludlow, M. M. Boyd, T. Zelevinsky, S. M. Foreman, S. Blatt, M. Notcutt, T. Ido, and J. Ye, Phys. Rev. Lett. 96, 033003 (2006); R. Le Targat et al., Phys. Rev. Lett. 97, 130801 (2006).

[12] Z. W. Barber, C. W. Hoyt, C. W. Oates, L. Hollberg, A. V. Taichenachev, and V. I. Yudin, Phys. Rev. Lett. 96, 083002 (2006).

[13] A. D. Ludlow et al., Science 319, 1805 (2008).

\section{About the Authors}

\section{Henning Moritz}

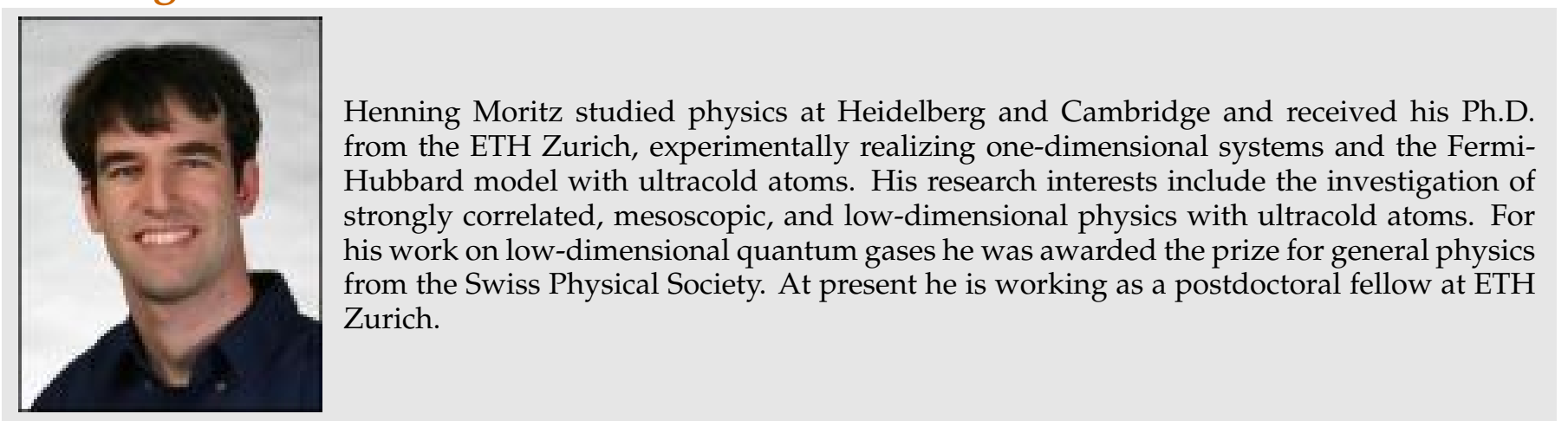

\section{Tilman Esslinger}

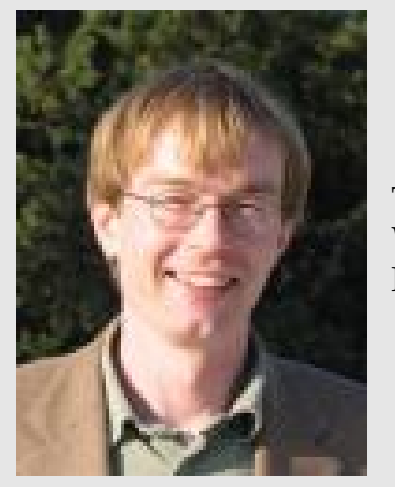

Tilman Esslinger studied physics at the University of Munich, Germany, and at the HeriotWatt University in Edinburgh, Scotland. He received his Ph.D. from the University of Munich and since 2001 has been full professor in the physics department of ETH Zurich. 